\title{
3D SKETCHES IN VIRTUAL REALITY AND THEIR EFFECT ON DEVELOPMENT TIMES
}

\author{
Seybold, Carsten; \\ Mantwill, Frank \\ Helmut Schmidt University Hamburg
}

\begin{abstract}
In the product development process, digital support continues to advance. Some work steps during product development are still carried out without assistance. Sketch creation is one of these. Therefore, the content created here is rarely documented due to the effort required for digital transformation. An alternative can be sketching in virtual reality. This article explores whether $3 \mathrm{D}$ sketching in VR enables faster sketching and can offer the basic features of hand-drawn sketches. To verify this, a tool for 3D sketching was developed. 27 test subjects were asked to solve one out of two different design tasks using this tool. The experiments were evaluated using video coding to identify the subjects actions. The created solutions have been analyzed about quality. The study showed initial indications that sketching in VR generally enables faster processing while maintaining the same solution quality.
\end{abstract}

Keywords: Virtual reality, Conceptual design, Embodiment design, Sketches

\section{Contact:}

Seybold, Carsten

Helmut Schmidt University Hamburg

Machine Parts \& Computer Aided Development

Germany

carsten.seybold@hsu-hh.de

Cite this article: Seybold, C., Mantwill, F. (2021) '3D Sketches in Virtual Reality and their Effect on Development Times', in Proceedings of the International Conference on Engineering Design (ICED21), Gothenburg, Sweden, 16-20 August 2021. DOI:10.1017/pds.2021.1 


\section{INTRODUCTION}

During the conceptual and embodiment design, product developers often use sketches or models to generate solutions. These sketches are often used to quickly visualise, develop and communicate concepts to clients and other product designers. According to Deisinger (2002), sketches in engineering design continue to be used to represent shapes, spaces, objects, proportions, technical functions, processes and relationships of objects. Goldschmidt (1991) sees the strengths of sketches in quick and fluid visualisation. Nik Ahmad Ariff et al. (2012) see sketches as a medium for understanding solutions and communicating ideas. Sachse and Leinert (2002) bring these different approaches to sketch definition together in a more comprehensive approach. They classify the sketch as an external support form. In addition to the sketch, simple models, production prototypes, tests or preliminary design drawings are also considered among the external forms of support. According to Sachse et al. (1999), the forms of support fulfil various functions. They are used as:

- $\quad$ analysis assistance to identify problems or weak points of the product at an early stage,

- evaluation assistance in order to be able to evaluate and secure solutions in particular,

- $\quad$ storage assistance (memory aid),

- $\quad$ solution assistance to generate new ideas,

- communication assistance and

- documentation assistance.

According to Sachse et al. (1999), sketches are frequently used for the stated functions, with the exception of use as an evaluation assistance. According to Hacker (1999), externalising thoughts in sketches relieves human short-term memory.

Consequently, sketches continue to be an important part of the product development process, as Ullman et al. (1990) confirmed in empirical studies. Hacker (1999) further confirms the importance of sketching. It is precisely the conceptualisation and consideration of fundamental solution options in the early stages of product development that has a decisive influence on the innovativeness of the solution and the production costs. According to Schütze et al. (2003), sketching in the early phases of product development leads to a demonstrably higher solution quality.

Even today, sketching is still done by hand. The cause, according to Römer and Pache (2002), is that the early phases of product development are not currently supported in a meaningful way by current computer-aided design (CAD) programmes. The use of hand sketches in contrast has proven itself since the early days of technical development and is used almost intuitively by developers. Despite these advantages for the developer, hand sketches in product development nevertheless have disadvantages. The most decisive point is the incompatibility of hand sketches with the subsequent digital product models. Furthermore, the creation of 2D views for three-dimensional objects of observation ties up the designer's mental capacities, which could ideally also be used for the search for solutions (Müller (2007)). As Pache (2005) already stated, a digital medium to support sketching should aim to cover the advantages of the classical way of working and compensate its deficits.

\section{RESEARCH APPROACH AND OBJECTIVE}

Therefore, it is necessary to identify a digital medium for sketching that allows improved compatibility with the digital systems used in the following process steps of product development. This applies firstly to authoring software such as CAD and secondly to cross-process systems such as product data management (PDM) as Huet et al. (2009) describes. Furthermore, it is concluded from the attachment of mental capacity through the creation of $2 \mathrm{D}$ views that the creation of 3D sketches should be enabled. At the same time, such a system needs to provide the advantages of the classic way of working, such as easy and intuitive operation.

3D sketching with virtual reality can offer one possibility. Chu et al. (2017) states that the sketching tool can influence the style of sketching and the sketching activities of designers. The objective of this approach is to empirically investigate the effects of the use of such a tool on the processing times of sketches, as well as the temporal distribution of designers' actions in engineering design. Hand sketches can provide functions of external support forms such as analysis or communication assistance. This study is intended to provide an initial assessment if these functions can be covered by digital 3D sketching as well. Furthermore, it is examined whether the quality of the solutions from 3D sketching differs from the quality of classically created solutions using hand sketches. In order to be 
able to investigate the above-mentioned aspects, a VR tool was developed which provides sketching and modelling functions. Before going into the experimental design, we will first briefly present an overview of the possible alternatives or related research.

\section{ALTERNATIVES FOR DIGITAL SKETCHING AND THE USE OF VR}

An alternative could be primarily the use of CAD software for sketching. However, Schütze and Ulbricht (2006) stated that freehand sketching is more effective than sketching with CAD in terms of efficiency and effectiveness. A possible reason for this is that mental capacity is tied up by the complexity of CAD systems, which reduces mental capacity for problem solving. According to Müller (2007), sketches are based on the principle of intentional inaccuracy. The importance of individual lines is low within sketches and there is room for change. This is contrary to the principles of CAD systems. These systems require usually precise specifications. Although they name $\mathrm{CAD}$ as a suitable medium for visualisation and communication, Robertson et al. (2009) also advise against sketching with CAD.

However, other approaches already exist. Evans and Aldroy (2016) investigated the use of tablet PCs to create 2D digital sketches compared to hand sketches. Camba et al. (2018) also investigated the benefits of digital sketching as an alternative to traditional sketching using a graphics tablet and software. The software Lift-Off by Jackson and Keefe (2016) is a research approach to support the digitisation of hand-drawn sketches. These approaches focus on the field of industrial engineering and two-dimensional techniques. Approaches for sketching in three-dimensional virtual environments are presented below. As CAD or other CAx systems also represent a type of virtual technology, it is important to define this technology before proceeding. Therefore, the immersive virtual techniques according to Burdea and Coiffet (1994) used in the following approach will be delimited from the nonimmersive techniques. Immersive techniques appeal to as many of the user's senses as possible and allow the user to immerse in the environment. How much the user is integrated into the virtual environment depends on the user's imagination. Burdea and Coiffet (1994) cite the user's ability to interact with the virtual environment as a third important aspect of VR. Head Mounted Displays (HMDs) and CAVE systems (projection-based VR environments) are classified as such immersive VR systems according to Dörner et al. (2013).

Of course, VR is already used in product development. The use of VR is mainly limited to the representation of visual product characteristics, as Rademacher (2014) points out. One approach to use VR for sketching by Pache (2005) elaborated which functionalities a 3D sketcher should provide the user. Deisinger (2002) focused on the tools for modelling and sketching techniques with the development of a hybrid modelling system. Ranscombe et al. (2019) investigated the use of Digital Sketch Modelling as a hybrid tool to complement sketching and CAD. The development of a hardware and software concept for future 3D sketching environments in VR is the focus of Fechter and Wartzack (2016). In addition, Fechter et al. (2020) have implemented CAD-like modelling in VR that focuses on the adaptation of existing geometry in the field of engineering design. Feeman et al. (2018) developed and investigated a CAD-like modelling tool in VR for industrial design and evaluated it in comparison to the use of traditional CAD software.

Amicis (2002) and Xin et al. (2008) used augmented reality (AR) for sketching in their research. While Amicis (2002) equipped AR with CAD functionalities for sketching in the hybrid virtual and real environment, Xin et al. (2008) developed a system that enables the creation of 3D representations via $2 \mathrm{D}$ sketches and projections and can be operated via tablet $\mathrm{PC}$.

In summary, there are numerous approaches, but the majority are related to industrial design. 3D approaches focus mainly on modelling geometry, less on sketching techniques or a mixture of both. The approach pursued here using a 3D sketching tool in VR can therefore complement the previous research approaches with further results.

\section{TEST PROCEDURE}

Using the VR tool, a group of test subjects had to solve different tasks in the area of engineering design. For this purpose, the test subjects were asked about their personal characteristics and their own experience in the field of product development and VR via questionnaires before starting the experiment. Subsequently, the subjects received a basic training in the use of VR and the VR processing tool, in form of a two-part tutorial. Following the gamification approach according to Kapp (2012), the use of the HMD and the controllers are taught in a playful manner. In the following, the 
functions of the tool are instructed solving an example task in VR. The training based on the tutorials is designed for completion within one hour.

The experiment began for the test subjects with the written provision of the task and can be divided into two sections. In the first section, the test subjects were given the opportunity to analyse the task and make comments. The supervisors were available to answer questions about the content, if necessary. When the test subject had finished the preparation, he or she could start working in VR. During this work, the test subject had the possibility to interrupt the work in VR at any time and look at the recordings or task. To minimise interruptions while working in the VR, the most important information from the design tasks was provided via several display boards inside VR. After the experiment, the test subjects were asked again about their personal assessment of their work in VR and the execution of the experiment via a questionnaire. In addition, the results were discussed in a debriefing session together with the experimental supervisor.

For evaluation, the solutions developed by the test subjects and the test processing itself were used. The experiments accompanied by two supervisors and have been recorded on video. The actions and statements of the test subjects were coded in the videos using a category system by two independent raters. The inter-rater reliability was about $\kappa=0.73$ (strong agreement, Brennan and Prediger (1981) and Landis and Koch (1977)). The category system can be separated into processing times and nonprocessing times. Among the processing times, there are several sub-categories for a more detailed analysis of the activities. The most important ones are briefly presented as follows:

- Sketching \& modelling: This category includes all sections in which the test subject executes practical activities such as creating or positioning geometry. This category also covers the preparation and follow-up activities necessary for these actions. This can be the selection of required sketching functions in the menu, for example.

- Verifying information: This category contains the codes in which the test subject checks information about the design task. This can be general information, such as the connection planning of hydraulic lines, or checking specific requirements.

- Checking possible solutions: This coding is used to cover sections in which the test subject visibly checks elements of his own solution or weighs up alternatives before creating new content. Looking at sketched elements from all sides is an example of this case.

- Explaining \& discussion about solutions: All sections in which the test subject talks to the supervisor about the solution or possible alternative solutions are covered with this coding. It is important to note that the supervisors neither suggested solutions nor evaluated the subject's solutions during the processing.

- Clarifying issues: If the test subject had questions about the design task during the video recordings, the sections were coded accordingly in this category.

Parallel to the processing sections, the sections of non-productive work have also been coded. These sections include processing breaks, such as saving work statuses, software \& operating errors and time the test subject spent for searching \& testing functions. Sections of restoring own solution after an error or unintentionally deleting content by the test subject, for example, were coded accordingly.

The experimental recordings were coded sequentially and continuously. If several codings were present at the same time, only the predominant category was coded due to the proportions of the total time.

In addition, a comparative subject worked on the design tasks by making hand-drawn sketches with pen and paper. The use of CAD was permitted for viewing the individual parts provided within the design task and their positioning.

In this study, the sketches developed by the test subjects were evaluated in addition to the previously determined times. Sevier et al. (2017) developed a solution-neutral approach to sketch evaluation using the factors complexity and clarity based on the methods of Rodgers et al. (2000) and Besemer (1998). This approach is suitable for different types of studies. Sevier et al. (2017) themselves suggest its use in sketching education. Nikolić et al. (2019) successfully applied the approach in the field of communication. For this study, it has been shown that an application is not feasible. Due to the conditions of the 3D sketch creation in the VR environment, a significant proportion of the rating factors could not be evaluated (e.g. shading, alternative views, texture, where, when). Other rating factors could only be evaluated in the same way (e.g. scale, 3D, annotations, kinetic) for all test subjects.

Therefore, we decide to use a solution-based evaluation according to the Cost-Benefit Analysis. Beitz et al. (2007) classify the Cost-Benefit Analysis as a procedure for the evaluation of complex products with a large number of evaluation criteria. The weighted requirements for the design were used as 
evaluation criteria. Two independent experts carried out the evaluation of the solution quality determined here.

\subsection{The design tasks}

The design tasks processed by the test subjects have their origin in real issues. The task descriptions were provided to the test subjects in written form and contained all the necessary information for processing. The information was provided in such a way that the test subjects had to work out and prioritise the requirements for their solutions. The objective for the test subjects was to develop a conceptual design sketch, which enables further work and detailing in CAD.

The objective in design task one was to develop a design sketch for an engine test bench. The test bench should accommodate a car engine including all necessary attachments and enable the operation of the engine on the test bench. The test subjects had to plan the test stand frame, the positioning of specified add-on parts on this frame, as well as the cooling and fuel lines in their sketch. In addition, the test subjects had to ensure that the test stand is mobile and fulfils other requirements. As a second task, a problem from the field of facility and workplace design had been used. The objective was to redesign an area of test benches. The test subject had to select suitable clamping fields, test benches and the associated valve and pump units and plan their arrangement. Escape and supply routes or the accessibility of the superstructures with a crane had to be ensured. A routing plan of the hydraulic lines completed the subject's tasks.

For the tasks all required 3D models of the components were provided to the test persons for use in VR in addition to the written information. These were, e.g. the car engine, the fuel pump or the radiator, which were to be accommodated in the test stand. For task two, Redesign of a servohydraulic test area, the range of models such as the test benches and valve blocks is extended via catalogue data to select suitable models for the preferred solution. These are clamping fields or tower cranes, for example.

\subsection{The 3D Sketching Tool}

There are already commercial software solutions for sketching in VR on the market. Some of these are gravity sketch or flyingshapes. These software applications already provide numerous sketching functions. Many functions are based on surface editing, because these tools focus on sketching for product styling or industrial design. However, sketching for engineering design is also possible in principle.

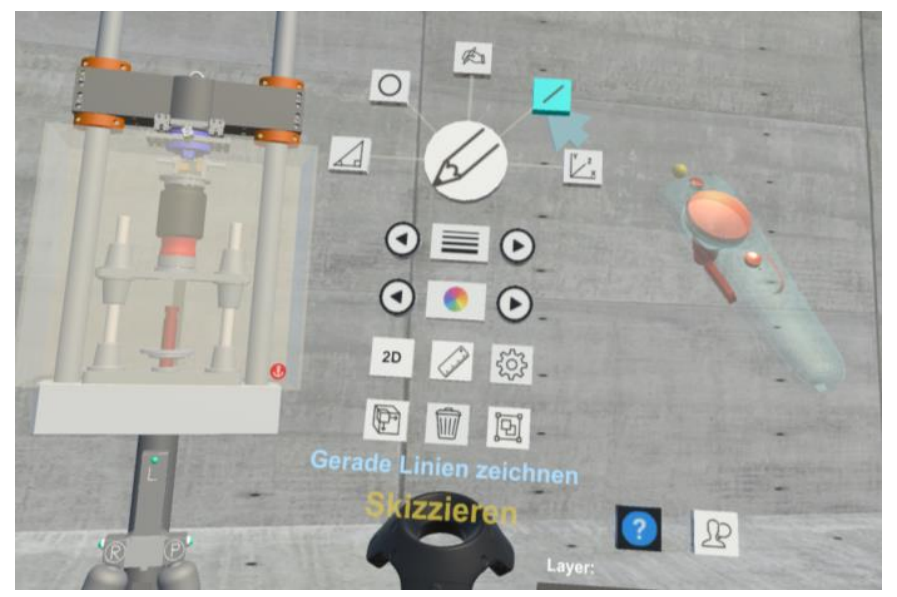

Figure 1. Sketching menu of the 3D sketching tool with straight-line function selected

However, the use of commercial software for the experiments was discarded for several reasons. In 3D sketching environments, it is possible to proceed differently when creating new content. On the one hand, it is possible to provide tools that enable drawing work, oriented towards the way of working with hand sketches. The user selects a tool and drawing parameters (such as line shape and thickness) and begins to create a line of the desired length, for example. On the other hand, it is also possible to work model-based using basic bodies. The user is offered prepared bodies from a modular system (e.g. cubes, cylinders) and can manipulate and change the dimensions of the objects as required. The subjects should be enabled to choose their own way of working. Commercial software is largely 
oriented towards only one way of working. The fast learnability of the handling of the tool, the use of numerous 3D models from CAD per design task and the necessity to be able to record the activities and results of the test subjects were further reasons for developing a separate environment for conducting the experiment.

The self-developed tool is based on the Unity development environment using the HMD HTC Vive. In addition to the actual HMD hardware, the system offers two controllers and a comparatively large movement space of $12 \mathrm{~m}^{2}$. The tool provides functions for drawing sketches and modelling via basic bodies. It is possible to mix both functional groups. The functions are made available to the user via a selection menu on one of the controllers. With the other controller, the user can select them and use them in the environment, as shown in Figure 1.

The sketching functions include freehand sketching, drawing straight lines or arcs, for example. Optionally, the line width, colour and line shape can be adjusted. If the user want to work partly in two dimensions, it is possible to work on auxiliary planes. Geometric basic bodies such as rectangles, spheres, cylinders, etc. are available as modelling basis. However, the use of principle symbols for simple machine elements like springs, dampers, roller bearings, etc. is also possible. The objects can be freely positioned in the virtual environment and they can be copied, grouped and manipulated in their dimensions. Further supporting tools are the measuring of distances and the insertion of audio comments via speech-to-text. The work progress can also be saved and loaded in the VR environment. An export via STL and FBX-format for further use, e.g. in CAD, is possible.

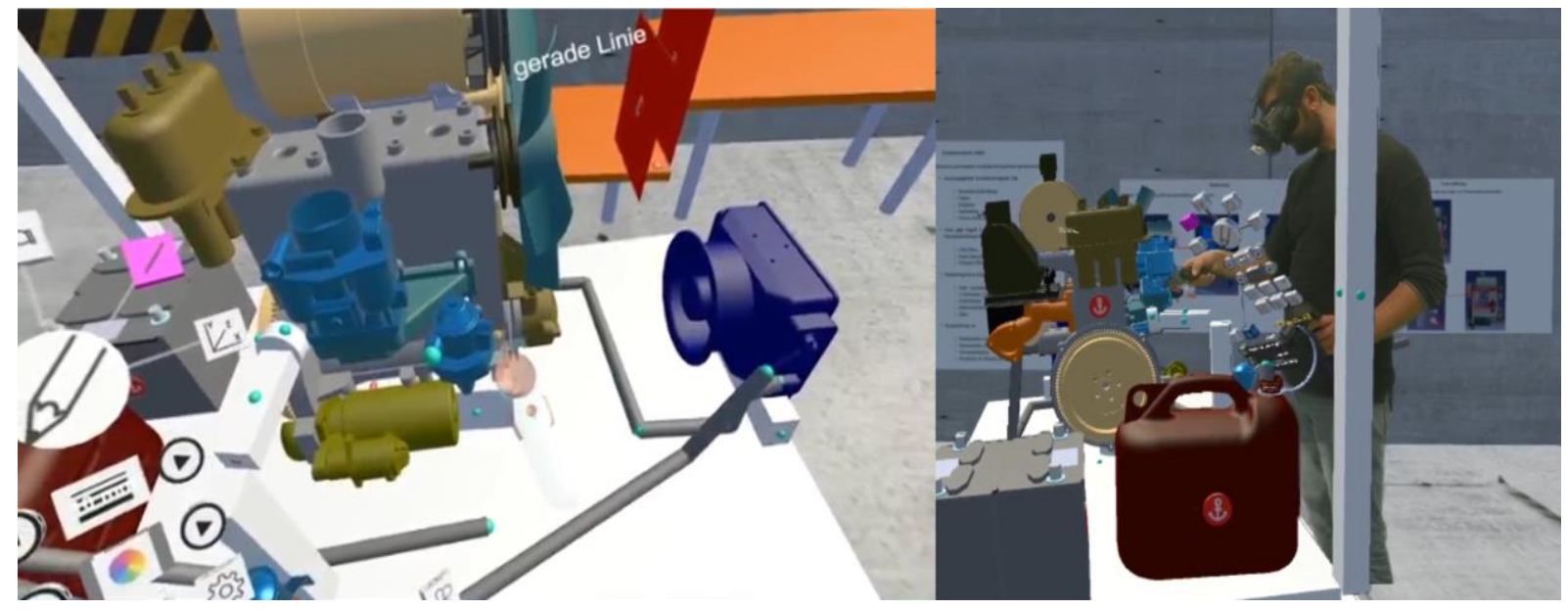

Figure 2. Test subject working on the task "Design of an engine test bench". Field of view on the left and mixed reality view on the right side

Furthermore, the task-specific content presented in the previous chapter is provided to the test subjects within the tool. For this purpose, the test subjects are offered a room for each task, in which the 3D models of the car engine, test benches, etc. and the test bench environment are available. The tool developed is of course only a prototype. However, it is designed in such a way that a processing time of 1-2 hours would be possible in the daily workflow of the product developer.

The test subjects were recorded during the processing by video via a Mixed Reality (MR) setup. This setup enables the recording of the real person's movements in the context of the virtual environment. A green screen and a third controller of the HTC Vive are used for this purpose. The controller is used to synchronize the position of the real camera and a virtual one in the virtual environment. As a result, the real view and virtual view are synced in the video. The field of view of the test subject is recorded for evaluation additionally. Both views are shown in Figure 2.

\subsection{Description of the sample}

Twenty-seven test subjects worked in VR on the tasks in total. Task one, Design of an engine test bench, has been processed by 15 test subjects and 12 test subjects worked on task two, Redesign of a servo-hydraulic test area. Twelve test subjects declared that they were product development students. All students have been imminent before the master degree at the time of the experiment. This group of test subjects had comparatively little professional experience in the field of product development $(\mu=$ 0.6 years; $\sigma=0.6$ years). Further 12 test subjects belonged to the occupational group of engineers with 
an academic degree. The average experience in product development is about $\mu=2.5$ years $(\sigma=2.2$ years). With three test subjects, a third occupational group without academic education can be formed. This group includes two educated CAD engineers and one engineering draughtsman. All of them work in product development every day. The professional experience of this group is much higher with an average of $\mu=15.7$ years ( $\sigma=4.9$ years). The random distribution of the subjects according to Dörner et al. (2013) is shown in Table 1.

Table 1. Distribution of the test subjects to the tasks

\begin{tabular}{lccc}
\hline task & students & technical engineers & CAD engineers/draftsmen \\
\hline Redesign of a servo-hydraulic test area & 8 & 3 & 1 \\
Design of an engine test bench & 4 & 9 & 2 \\
\hline
\end{tabular}

In front of the experiment, the test subjects stated in the questionnaires that they only had a low level of experience with VR $(\mu=1.70, \sigma=0.91$; value range from 1 (low) to 5 (high)). The subjects rated their skill in sketching as medium $(\mu=3.11, \sigma=0.89)$. However, the subjects rated the importance of sketches or models before working in CAD as medium to high $(\mu=4.00, \sigma=0.78)$.

An independent test subject with 30 years of professional experience as a designer has drawn up a comparative solution for each task. These solutions include hand drawn sketches with the partial aid of CAD for component positioning in one case. The subject solved both tasks.

\section{EVALUATION OF THE RESULTS}

The test results can be found in Table 2 and 3 for both tasks. The time taken by the subjects to complete the analysis of the task before working in VR has been recorded. Main activities in this part of the experiment were reading the task and writing down notes. For this activity, no difference was expected between the test series in VR and the reference subject. For the task Design of an engine test bench, the reference subject was even significantly faster.

Table 2. Results for the task 'Redesign of a servo-hydraulic test area'

\begin{tabular}{llll}
\hline & VR test subjects & reference test subject & significance \\
\hline \multirow{2}{*}{ Task analysis } & $\mu=1: 03: 52 \mathrm{~h}$ & \multirow{2}{*}{$0: 56: 21 \mathrm{~h}$} & not significant \\
& $\sigma=0: 18: 18 \mathrm{~h}$ & & $t(11)=1.4, p=0.181^{1}$ \\
Sketching time & $\mu=0: 45: 03 \mathrm{~h}$ & \multirow{2}{*}{$1: 31: 22 \mathrm{~h}$} & significant \\
& $\sigma=0: 10: 36 \mathrm{~h}$ & & $t(11)=-15.1, p<0.001$ \\
\multirow{2}{*}{ Solution quality } & $\mu=0.84$ & 0.71 & significant \\
& $\sigma=0.05$ & & $t(11)=8.6, p<0.001$ \\
\hline
\end{tabular}

${ }^{1}$ One-Sample t-Test, $\alpha=0.05$

The sketching times are examined next. The test subjects in VR were significantly faster than the reference subject in both tasks. If the sketch processing and the analysis of the design task are observed together, a significantly faster working in VR still results for both tasks. In the next step, it is necessary to check whether the solutions created in VR can also offer the same solution quality as the conventionally created solutions. According to Table 3 , there is no significant difference between the design solutions of the VR test subjects and the reference test subjects for the task Design of an engine test bench. Table 2 reveals that for the task Redesign of a servo-hydraulic test area a significantly higher solution quality exists for the solutions created in VR. Some selected solutions with different solution quality are shown at the end of the paper in Figure 4.

Table 3. Results for the task 'Design of an engine test bench'

\begin{tabular}{|c|c|c|c|}
\hline & VR test subjects & reference test subject & significance \\
\hline Task analysis & $\begin{aligned} \mu & =0: 46: 12 \mathrm{~h} \\
\sigma & =0: 22: 56 \mathrm{~h}\end{aligned}$ & $0: 31: 45 h$ & $\begin{array}{l}\text { significant } \\
t(14)=2.4, p=0.029^{1}\end{array}$ \\
\hline Sketching time & $\begin{aligned} \mu & =0: 43: 11 \mathrm{~h} \\
\sigma & =0: 09: 41 \mathrm{~h}\end{aligned}$ & $2: 54: 38 h$ & $\begin{array}{l}\text { significant } \\
t(14)=-52.6, p<0.001\end{array}$ \\
\hline Solution quality & $\begin{aligned} \mu & =0.78 \\
\sigma & =0.08\end{aligned}$ & 0.81 & $\begin{array}{l}\text { not significant } \\
t(14)=-1.1, p=0.286\end{array}$ \\
\hline
\end{tabular}

${ }^{1}$ One-Sample t-Test, $\alpha=0.05$ 
After the test, the subjects rated the work with VR positively in the questionnaire ( $\mu=1.52, \sigma=0.70$; with a range of values from -2 (strongly disagree), via 0 (neither disagree nor agree) to 2 (strongly agree)). In detail, the test subjects stated that both the training via the tutorial $(\mu=1.81, \sigma=0.40)$ and the VR processing application $(\mu=1.42, \sigma=0.64)$ were sufficient for carrying out the experiment.

In the following, the individual categories of processing times will be discussed. The average times from the video coding can be found in Figure 3. The average times for the coded sections for verifying information and checking possible solutions are a significant part of the processing time. They took across both design problems an average of $\mu=5: 33 \mathrm{~min}$ (19\% of the processing time) and $\mu=4: 06 \mathrm{~min}$ $(14 \%)$ in total. This is a first indication that sketches in VR can also be used as analysis assistance.

Only a very limited statement can be made about the usefulness of $3 \mathrm{D}$ sketches in VR as a communication assistance by analysing the time periods. Due to the fact that the tasks were to be solved by the subjects as individual work as well as the neutrality of the supervisors, discussions about alternative solutions could only be held to a very limited extent. In addition, the test subjects were asked to explain their solution at the end of the experiment. The average time for explaining \& discussion about solutions of $\mu=4: 28$ min with a part of $15 \%$ of the processing time can therefore only be seen as a tendency. On average $\mu=2: 34 \mathrm{~min}$ are allocated to the explanation at the end of the experiment. However, the used time for clarifying issues with an average of $\mu=2: 51 \mathrm{~min}$ and a part of about $9 \%$ of the processing time provides indications that 3D sketches in VR could also be suitable as a communication assistance.

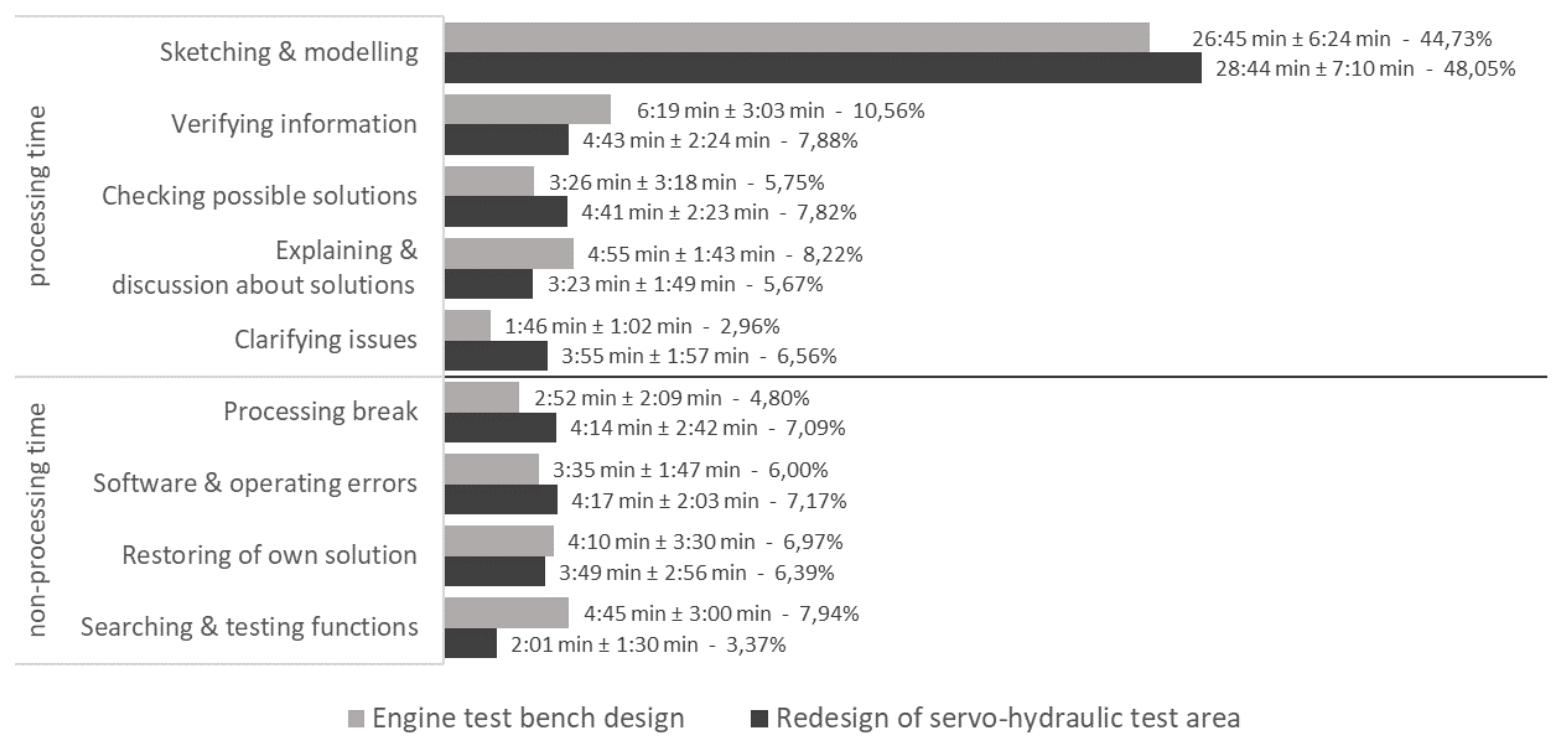

Figure 3. Average times and standard deviation for the coded activities of both test series

Examining the total time of the experiments in VR, it is evident that the non-processing times take up a high proportion (25\%). This can be explained by two aspects. On the one hand, the VR sketching tool is not a market-ready software, so software errors are possible. On the other hand, the test subjects were only trained for the VR environment between $60 \mathrm{~min}$ to $90 \mathrm{~min}$. This is naturally not comparable with extensive training, which is usual with CAD use, for example. With the use of commercial software and with extensively trained users, it can be assumed that these proportions will decline strongly in practice.

\section{CONCLUSION}

A critical examination of the results remains necessary. The small sample size, only two different tasks and only one reference test subject are factors that influence the significance of the results. The results presented here can therefore only provide an initial orientation and are the basis for further studies. The implementation of further studies with larger sample sizes and a larger control group should therefore be aimed for. However, the results show that the use of 3D sketches in VR provides initial indications of faster sketch creation with the same solution quality. It is remarkable that the test subjects were able to generate comparable solution qualities in VR as the reference test subject, although the average work experience is significantly lower due to the high proportion of students. It would therefore be 
conceivable in the future to release sketch creation from the product developer's field of activity and to carry it out into collaboration with less trained personnel e.g. product customers.

Basically, the individual times of the categories show that 3D sketching in VR also includes functions of external forms of support. However, considering these times separately is not sufficient to make valid statements, e.g. about the extent and type of analysis or communication support. Here, further studies are necessary as well. This study shows the potential of sketching in VR. Based on this, further studies regarding to clarity of the solutions, suitability for assessment, constructive reusability of the solutions and other factors such as the number of design changes are carried out in order to achieve a general overview in the context of a case study according to Yin (2018).

Furthermore, the process-related and technical integration of 3D sketching in VR into the product development process was not considered in this paper. There is, among other things, a need for further action with regard to the quality of the 3D sketch models and their possible further use. The processrelated integration of 3D sketches in PDM systems is part of further research.

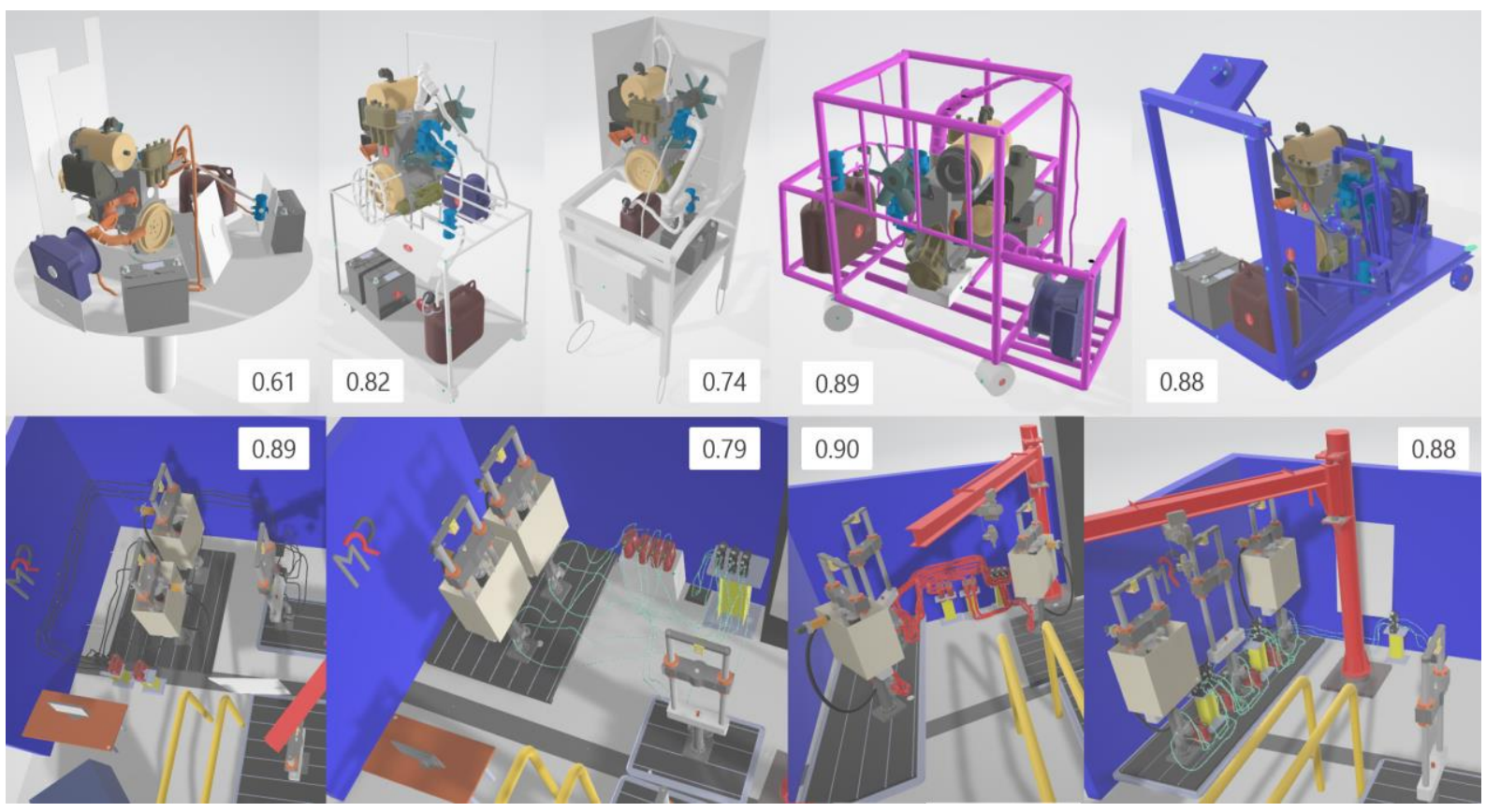

Figure 4. Various examples of solutions from the subjects including solution quality. Task one above, task two below

\section{REFERENCES}

Amicis, R. (2002), "SketchAR : Sketching in Mixed Realities", In: Gausemeier, J. (Ed.), Paderborner Workshop Augmented \& Virtual Reality in der Produktentstehung, pp. 145-156.

Besemer, S.P. (1998), "Creative product analysis matrix: Testing the model structure and a comparison among products - Three novel chairs", Creativity Research Journal, Vol. 11, pp. 333-346.

Brennan, R.L. and Prediger, D.J. (1981), "Coefficient Kappa: Some Uses, Misuses, and Alternatives", Educational and Psychological Measurement, Vol. 41 No. 3, pp. 687-699.

Burdea, G. and Coiffet, P. (1994), Virtual reality technology, Wiley, New York.

Camba, J.D., Kimbrough, M. and EunSook, K. (2018), "Conceptual product design in digital and traditional sketching environments: a comparative exploratory study", Journal of Design Research, Vol. 16, pp. 131154. https://doi.org/10.1504/JDR.2018.10014195.

Chu, P.Y., Hung, H.Y., Wu, C.F. and Liu Y.T. (2017), "Effects of various sketching tools on visual thinking in idea development", International Journal of Technology and Design Education, Vol. 27, pp. 291-306. https://doi.org/10.1007/s10798-015-9349-5

Deisinger, J. (2002), Entwicklung eines hybriden Modelliersystems zur immersiven konzeptionellen Formgestaltung, Jost-Jetter, Heimsheim.

Dörner, R., Broll, W., Grimm, P. and Jung, B. (2013), Virtual und Augmented Reality (VR / AR): Grundlagen und Methoden der Virtuellen und Augmentierten Realität, Springer, Heidelberg. http://doi.org/10.1007/9783-642-28903-3

Evans, M. and Aldoy, N. (2016), "Digital design sketching using the tablet PC", The Design Journal, Vol. 19 No. 5, pp.763-787. 
Fechter, M. and Wartzack, S. (2016), "Konzept für ein VR-System zur intuitiven Modellierung durch natürliche Interaktion”, In: Stelzer, R. (Ed.), Beiträge zur virtuellen Produktentwicklung und Konstruktionstechnik, pp. 561-570.

Fechter, M., Schleich, B. and Wartzack, S. (2020), "CAD-Gestaltmodellierung in VR für die frühe Entwurfsphase", Konstruktion, Vol. 72, pp. 69-74.

Feeman, S.M., Wright, L.B. and Salmon, J.L. (2018) "Exploration and evaluation of CAD modeling in virtual reality", Computer-Aided Design \& Applications, Vol. 15 No. 6, pp. 892-904.

Goldschmidt, G. (1991), "The dialectics of sketching", Creativity Research Journal Vol. 4 No. 2, pp. $123-143$.

Hacker, W. (1999), "Konstruktives Entwickeln als Tätigkeit: Versuch einer Reinterpretation des Entwurfsdenkens (design problem solving)" Zeitschrift für Sprache und Kognition, Vol. 18 No. 3/4, pp. 88-97.

Huet, G., McAlpine, H., Camarero, R., Culley, S.J., Leblanc, T. and Fortin, C. (2009), "The Management of Digital Sketches through PLM solutions", In: Proceedings of ICED 09, the 17th International Conference on Engineering Design, Vol. 8, pp. 239-250.

Jackson, B. and Keefe, D.F. (2016), "Lift-Off: Using Reference Imagery and Freehand Sketching to Create 3D Models in VR", IEEE Transactions on Visualization and Computer Graphics, Vol. 22 No. 4, pp. 1442-1451.

Kapp, K.M. (2012), The gamification of learning and instruction: Game-based methods and strategies for training and education, Pfeiffer, San Francisco.

Landis, J.R. and Koch, G.G. (1977), "The Measurement of Observer Agreement for Categorical Data", Biometrics, Vol. 33 No. 1, p. 159. http://doi.org/10.2307/2529310

Müller, F.D. (2007), Intuitive digitale Geometriemodellierung in frühen Entwicklungsphasen, Dr. Hut, München.

Nik Ahmad Ariff, N.S., Badke-Schaub, P., Eris, O. and Suib, S.S.S. (2012), “A framework for reaching common understanding during sketching in design teams", Proceedings of the 12th International Design Conference (DESIGN 2012), Dubrovnik, Croatia, pp. 1525-1533.

Nikolić, M., Škec, S., Martinec, T. and Horvat, N. (2019), "The Role of Sketching Activities and Outcomes in Conceptual Design Phase", In: Proceedings of the 22nd International Conference on Engineering Design (ICED19), Delft, The Netherlands, 5-8 August 2019. http://doi.org/10.1017/dsi.2019.43

Pache, M.W. (2005), Sketching for conceptual design: Empirical results and future tools, Dr. Hut, München.

Pahl, G., Beitz, W., Blessing, L., Feldhusen, J., Grote, K. and Wallace, K. (2007), Engineering Design: A Systematic Approach, Springer, London. http://doi.org/10.1007/978-1-84628-319-2

Rademacher, M.H. (2014), Virtual Reality in der Produktentwicklung: Instrumentarium zur Bewertung der Einsatzmöglichkeiten am Beispiel der Automobilindustrie, Springer, Wiesbaden.

Ranscombe, C., Zhang, W., Rodda, J. and Mathias, D. (2019), "Digital sketch modelling: proposing a hybrid visualisation tool combining affordances of sketching and CAD", Proceedings of ICED 19, the 22nd International Conference on Engineering Design, pp. 309-318. http://doi.org/10.1017/dsi.2019.34

Robertson, B.F. and Radcliffe, D.F. (2009), "Impact of CAD tools on creative problem solving in engineering design”, Computer-Aided Design, No. 41, pp. 136-146.

Rodgers P. A., Green, G. and McGown, A. (2000), "Using concept sketches to track design progress", Design Studies, Vol. 21 No. 5, pp. 451-464. https://doi.org/10.1016/S0142-694X(00)00018-1

Römer, A. and Pache, M. (2002), "Skizzieren und Modellieren in der Produktentwicklung: Hilfsmittel des Praktikers auch bei CAD-Arbeit?" In: Hacker, W. (Ed.), Denken in der Produktentwicklung: Psychologische Unterstützung der frühen Phasen, Hampp, Zürich, S. 53-62.

Sachse, P., Hacker, W., Leinert, S. and Riemer, S. (1999), "Prototyping als Unterstützungsmöglichkeit des Denkens und Handelns beim Konstruieren”, Zeitschrift für Arbeits- und Organisationspsychologie, No. 43, pp. 71-82.

Sachse, P. and Leinert, S. (2002), "Skizzen und Modelle - Wieso Hilfsmittel des Denkens und Handelns beim Konstruieren?”, In: Hacker, W. (Ed.), Denken in der Produktentwicklung: Psychologische Unterstützung der frühen Phasen, Mensch, Technik, Organisation, Hampp, Zürich, pp. 63-82.

Schütze, M., Sachse, P. and Römer, A. (2003), "Support value of sketching in the design process." Research in Engineering Design, Vol. 14 No. 2, pp. 89-97. https://doi.org/10.1007/s00163-002-0028-7

Schütze, M. and Ulbricht, S. (2006), "Einsatz verschiedenartiger Arbeitsmittel in den frühen Phasen der Produktentwicklung - Eine ergebnis- und prozessorientierte Analyse", In: Zeitschrift fur Arbeits- und Organisationspsychologie, Vol. 50 No. 2, pp. 79-91. https://doi.org/10.1026/0932-4089.50.2.79

Sevier, D.C., Jablokow, K., McKilligan, S., Daly, S.R., Baker, I.N. and Silk, E.M. (2017), "Towards the development of an elaboration metric for concept sketches", In: Proceedings of the ASME Design Engineering Technical Conference, Vol. 3. https://doi.org/10.1115/DETC2017-67375

Ullman, D. G., Wood, S. and Craig, D. (1990), "The importance of drawing in the mechanical design process", In: Computers and Graphics, Vol. 14 No. 2, pp. 263-274.

Yin, R.K. (2018), Case study research and applications: Design and methods, Sage, Los Angeles.

Xin, M., Sharlin, E. and Costa Sousa, M. (2008), "Napkin Sketch - Handheld Mixed Reality 3D Sketching", In: Proceedings of the 2008 ACM symposium on Virtual reality software and technology, pp. 223-226. 